\title{
Design and First Tests of the CDF Run 2 Farms
}

\author{
Jaroslav Antos ${ }^{\mathrm{a}, \mathrm{c}}$, M. Breitung ${ }^{\mathrm{b}}$, Tony Chan ${ }^{\mathrm{c}}$, Paoti Chang ${ }^{\mathrm{c}}$, \\ Yen-Chu Chen ${ }^{c}$, Troy Dawson ${ }^{b}$, Jim Fromm ${ }^{b}$, Lisa Giacchetti ${ }^{b}$, \\ Tanya Levshina ${ }^{b}$, Igor Mandrichenko ${ }^{b}$, Ray Pasetes ${ }^{b}$, \\ Marilyn Schweitzer ${ }^{b}$, Karen Shepelak ${ }^{b}$, Miroslav Siket d,c, \\ Dane Skow ${ }^{b}$, Stephen Wolbers ${ }^{b}$, G.P.Yeh ${ }^{b}$, Ping Yeh ${ }^{c}$ \\ ${ }^{a}$ Institute of Physics, Slovak Academy of Sciences, Slovak Republic \\ ${ }^{\mathrm{b}}$ Fermi National Accelerator Laboratory, Batavia, IL, USA \\ ${ }^{\mathrm{c}}$ Academia Sinica, Taiwan \\ ${ }^{\mathrm{d} C o m e n i u s ~ U n i v e r s i t y, ~ S l o v a k ~ R e p u b l i c ~}$
}

\begin{abstract}
The high energy physics experiment CDF, located in the anti-proton-proton collider at Fermilab, will write data in Run 2 at a rate of $20 \mathrm{MByte} / \mathrm{s}$, twenty times the rate of Run 1. The offline production system must be able to handle this rate. Components of that system include a large PC farm, I/O systems to read/write data to and from mass storage, and a system to split the reconstructed data into physics streams which are required for analysis. All of the components must work together seamlessly to ensure the necessary throughput. A description will be given of the overall hardware and software design for the system. A small prototype farm has been used for about one year to study performance, to test software designs and for the first Mock Data Challenge. Results from the tests and experience from the first Mock Data Challenge will be discussed. The hardware for the first production farm is in place and will be used for the second Mock Data Challenge. Finally, the possible scaling of the system to handle larger rates foreseen later in Run 2 will be described.
\end{abstract}

PACS codes and keywords: 07.05.Bx, 07.05.Kf, 13.85.-t, Data analysis, data management, high-energy physics

\section{Introduction}

The CDF detector will take enormous amounts of data in Collider Run 2, which will begin in 2001 and will continue for 5 or more years. The Higgs and 
SUSY searches, among others, will require that data be analyzed as quickly as possible. To keep up with data taking, the data production will use large amounts of CPU power; therefore a large offline farm of computers is required. CDF and Fermilab have decided to use as much commodity hardware as possible to build the farm to reduce the cost. The choice is to build systems composed of hundreds of off-the-shelf PCs running the Linux operating system.

The main usage of the offline farm is for data production in quasi-real time, which includes reconstructing raw data, splitting the resulting output files into physics datasets and storing physics data on tape for physicists to analyze. It will also be used for data reprocessing. Monte Carlo generation is also likely.

This paper is organized as follows: In section 2 we state various requirements about Run 2 data and how they affect the farms. Section 3 shows the conceptual model of the farm that was built upon the facts and requirements. The efficiency of the farm is an important factor for the success of data production, thus it is the main focus when designing the production software system. Many factors contribute to the efficiency: error rate, error recovery time, idle time, etc. Their effects on the efficiency, as well as many other aspects of the system, are analyzed in section 4. Initial use of the software and the prototype farm hardware is given in section 5. A discussion of possible extension of capability beyond initial Run 2 rates is given in section 6 . Conclusions and next steps are given in section 7 .

\section{Requirements and Goals}

The major requirements and goals of the CDF production farms are the following:

- Rate and I/O Capacity

The capacity required for the farms is driven by the rate events are to be accepted and stored by the experiment and by the time it takes to reconstruct each event. Additional time will have to be added to handle reprocessing, Monte Carlo, splitting and any other anticipated needs.

The required average processing rate of the farms is $28 \mathrm{~Hz}$, based on a peak output rate from the experiment of $75 \mathrm{~Hz}$ and factoring in all the known downtimes. Given the input event size of 250 Kbytes the peak input rate is $20 \mathrm{MByte} / \mathrm{s}$. For output, the throughput requirement is $11 \mathrm{MByte} / \mathrm{s}$, given the ratio between output and input event sizes.

The CPU estimates (given in $500 \mathrm{MHz}$ PIII processor units) are 5 seconds per event which translates into a requirement of $140 \mathrm{PIII} / 500$ processors if the system runs at $100 \%$ efficiency. Choosing a more realistic $70 \%$ overall 
efficiency one calculates a requirement of $200 \mathrm{PIII} / 500$ processors to keep up with the DC rate. This does not include reprocessing, peak demands, Monte Carlo, etc. Therefore it is expected that the Run 2 CDF farm will be required to have approximately 300-400 PIII/500-equivalent processors. This translates to 6180-8240 SpecInt95.

- Ability to easily configure farm processing to control priorities.

The ease of use requirements include the ability to control priorities on the farms. This is important to allow the farm to be configured quickly to handle rapidly changing needs of the experiment. There may be new triggers which need to be understood and tuned, physics results which require rapid processing, or other conditions under which the farm has to be configured to process specified sets of data using certain code and constants.

- Excellent bookkeeping.

The bookkeeping that is required on the farms includes tracking the details of versions of code, constants, etc. which were used as well as a clean and clear way to correlate events or files with those version numbers. It also may include the capability to handle various requests about the number of events run through each version of the reconstruction package, etc. The exact information which needs to be kept will be driven by the needs of the people who run the farms, as well as the needs of the physicists who will analyze the data.

- Excellent error handling and error prevention.

One additional goal of such a system is to have excellent error handling. This makes such a system much easier to run and to maintain, and good attention to this will pay off over the long run. Also desirable is a welldesigned system in which errors are less likely to occur. In other words, attention will be paid to the experience of Run I farms and other related experiences as the system is designed.

\section{Conceptual Model}

The hardware of the data production system consists of:

- One or a few input/output nodes which are connected to the mass storage system with large disk buffers, and

- many worker nodes for data reconstruction.

The I/O node(s) and the worker nodes are connected by the network. The number of input nodes and output nodes are not yet specified.

It is expected that the system that processes new raw data will function as follows: The raw data files, stored in several input streams, reside on the mass storage system or external disks. A process stages raw data to disks 
on the input node. Each raw data file is then dispatched to one worker node for reconstruction. The reconstructed inclusive data along with monitor files (log files, bookkeeping records, validation histograms, etc) are collected to an output node. Inclusive data are split based on triggers into different physics datasets and concatenated to meet the 1 GB target file size. The physics data are then spooled to tapes. Some physics data may be copied to external disks. The monitor files are exported to an external production monitor computer for shift-takers to examine.

A conceptual model of the production system is shown graphically in figure 1 and it shows the data flow in the system.

We'll analyze this conceptual model in section 4.

\section{System Analysis}

All tasks in the system as well as the system as a whole are analyzed to arrive at a software design to fulfill the requirements and goals.

\subsection{Pipeline and Buffers}

The data production system can be viewed as a 6-stage data pipeline (input, dispatch, reconstruction, collect, split/concatenate, output), plus peripheral input/outputs. But it is not a usual straight-forward pipeline because:

(1) the parallel processing of worker nodes breaks the sequence of files, especially when errors occur; and

(2) the staging and spooling stage expect a collection of files as input while other stages operate with one file at a time.

It is mandatory to have data buffers at the input end of the split/concatenation and spool stage to accumulate files until they can be processed. The size of the data buffers is estimated to be 200 GByte for input staging, 200 GByte for output staging and 750 GByte for physics dataset output staging.

\subsection{Parallel Processing and Synchronization}

Synchronization is one main focus of the system because if the system is out of sync buffer filling may occur and the efficiency may be seriously degraded. 


\section{Conceptual Model of Run 2 Production System}

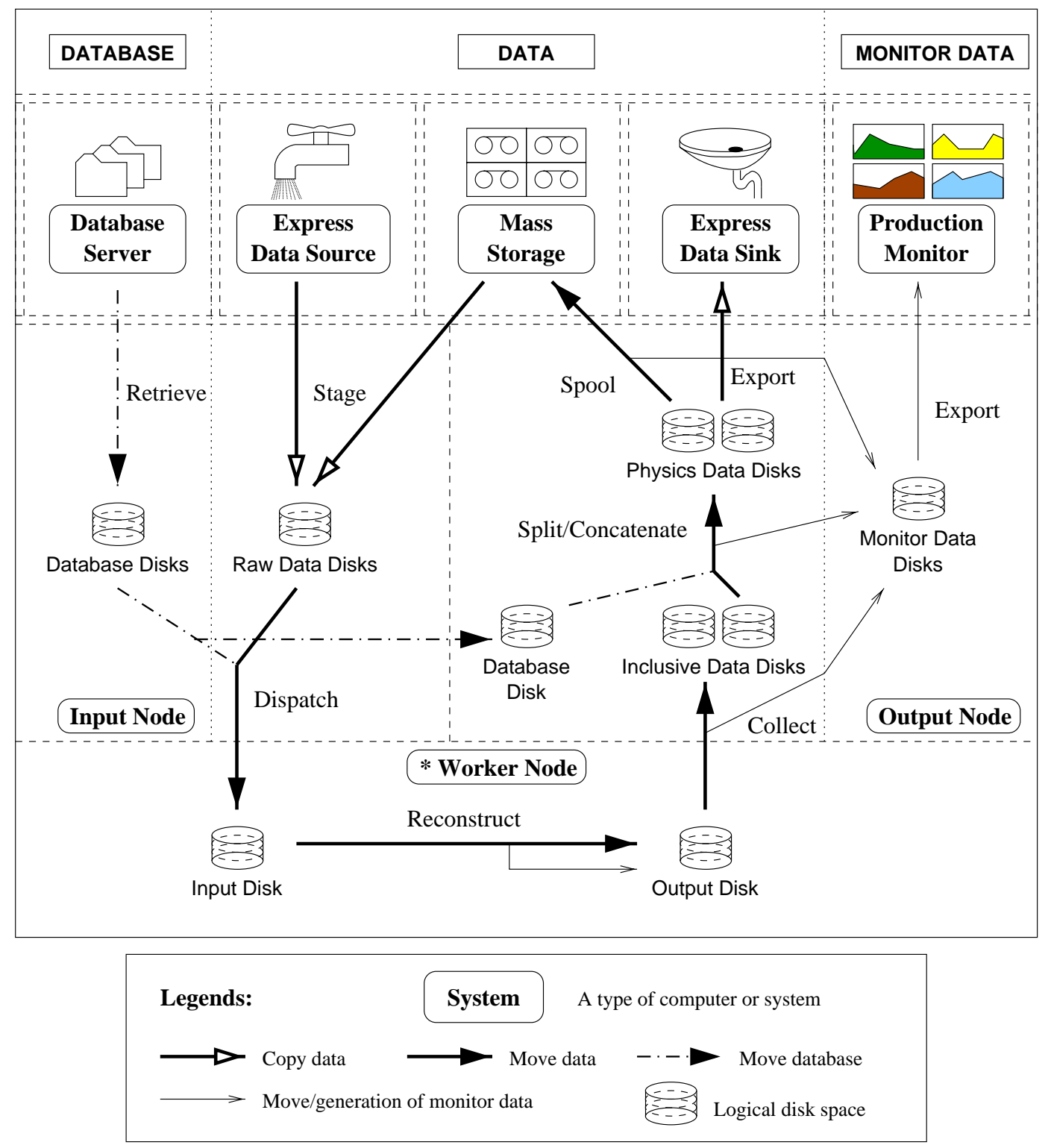

Fig. 1. The conceptual model of the Run 2 data production software system. External systems are enclosed in double dashed lines. Internal systems are separated by single dashed lines. Note that the separation between input node and output node is only logical. They could be the same physical computer.

Synchronization between raw data and calibration/trigger database on worker nodes is important. To avoid raw data files stacking up on worker nodes when the database is out of sync, it may be desirable to move this synchronization ahead to earlier stages in the pipeline. Since all disk space inside the production system is limited while the mass storage can be considered infinite in capacity, the best way is to trigger the "Input" activity for given raw data files only when corresponding databases are ready. This minimizes the probability of raw data disk congestion and/or waste of CPU time. 
The output of the split/concatenation stage are data of various physics datasets. Since the data of the same dataset aren't spooled to tape until a tape-worth (or possibly a file-set, approximately 10 GByte) of them appear on the disk, there is no clever solution for reducing the chance of congestion on physics disks. To avoid disk congestion, at least one of the following must hold:

(1) there are not too many physics datasets,

(2) the tape size is small,

(3) the physics data buffer is very large.

At this point it seems that option 3 is preferred.

The total throughput of a pipeline is determined by the slowest stage. Therefore we only need to analyze the rate and efficiency of each stage, and identify the slowest one.

The bandwidth of various parts of the production system are listed below:

- Tape drive:

The tape drive hardware is not decided. Before the start of Run 2 it is quite likely that newer technology with $6 \mathrm{MB} / \mathrm{s}$ speed or higher with capacity around $50 \mathrm{~GB}$ will mature. In that case we'll need 5 input tape drives and 3 output tape drives, plus a few more for buffering and spare.

- Disk:

The disk speed from an early test was approximately $20 \mathrm{MB} / \mathrm{s}$. With RAID disks, the disk speed is expected to reach $50 \mathrm{MB} / \mathrm{s}$ or more. However, it will drop considerably if multiple read/write occurs on the same disk array whether or not the disk head contention costs much performance. Therefore it may be desirable to avoid multiple read/write on the same physical disks.

- I/O bus:

The fibre channel on input/output nodes should have bandwidth around $1 \mathrm{~Gb} / \mathrm{s}$, or $\geq 90 \mathrm{MB} / \mathrm{s}$. The SCSI bus on the worker nodes will probably have bandwidth of $20-40 \mathrm{MB} / \mathrm{s}$. The bandwidth on worker nodes is not a problem because there are multiple worker nodes. The bandwidth on the output node is also suitable to sustain 5 concurrent read/write tasks of $11 \mathrm{MB} / \mathrm{s}$ each.

- Network:

The network on input/output nodes will be gigabit Ethernet $(\approx 90 \mathrm{MB} / \mathrm{s}$ wire speed), and on worker nodes are 100 BaseT $(\approx 9 \mathrm{MB} / \mathrm{s})$. With multiple worker nodes the bandwidth is sufficient.

Overall there are known no show-stoppers on bandwidth issues. 


\subsection{Worker Node Management and Utilization}

The Farms Group of the Fermilab Computing Division has been developing the Farm Batch System (FBS)(1) with the concept of "clean" worker nodes built in - all input files and output files can be deleted automatically when a job is done. It also provides worker node monitoring, among other features.

With FBS, one can submit job scripts in advance. FBS will start the job on a processor when the processor is available, and perform clean-ups after the job is done. In the job script, one can copy the file from an input node to a worker node, run the executable, and copy the files to an output node.

One caveat of this scheme is that it couples network input/output with CPU, so the CPU is idle when the worker node is doing network I/O. This increases the idle time. However, we expect the idle time to be fairly small because

(1) It takes about 8 hours to reconstruct a file, but it only takes about 3 minutes to copy it on input and output combined. That contributes $0.6 \%$ to the idle time.

(2) The input/output node will allow more than one network copy at the same time (limited by the disk head contention and network speed). That decreases the chance that the worker node has to wait.

\subsection{Bookkeeping and Catalog}

When data files go through the production pipeline, a bookkeeping database will be used to keep track of the data files that are in the system. The database may not need to be implemented with a commercial database system, but it has to allow reliable multiple access and remote access since both input node and output node will perform reading/writing.

Another database may be used to keep track of errors/failures for further error analysis. It is not known yet what the catalog system will be for the files exported to the external systems, another database may be needed to keep track of exported files.

\subsection{Error Handling}

Error handling is extremely important in such a large and dynamic system. It is not sufficient to fix problems "by hand", as the detailed work involved could potentially overwhelm the people involved and the farm could become idle for 
substantial fractions of time. Both of these situations are to be avoided.

The first level of error handling should be to avoid errors whenever possible. This will be built into the systems that comprise the farm system. It is hoped that the CDF executable will be robust. The farms software itself, including FBS, will be as error-free as possible. Finally, the hardware should be purchased with the avoidance of errors in mind. This may mean such simple things as specifying proper cooling in the PC's, redundant hardware or hot-swap capability, etc.

However, there will be errors at some rate and the next level of error handling will be the ability to automatically detect errors and take appropriate action. This can take many forms. If a worker node crashes, the reconstruction job could be automatically moved to another worker node, updating all of the logging databases appropriately. If the CDF executable crashes, the job could automatically restart. After a certain number of repeated failures the job could restart but skip the offending event (keeping a record of what was done of course). There are other approaches that could be taken. Other problems such as tape drive problems, disk failures, disks filling, etc. all should be handled automatically as much as possible.

The next level of error handling involves getting people ("shifters") to look at the system and take action to fix the problem, freeing disk space, calling operations to get systems reset, or whatever is required to get the farm running properly. The shifters could be paged automatically, be sent email, or be contacted when they monitor the farm as part of their shift responsibilities.

The higher levels of error handling involve experts and could involve rebooting machines, replacing bad hardware, helping to debug scripts or other control software, contacting CDF code experts, etc. This level of error handling should be invoked rarely in a smoothly operating system.

\section{$4.6 \quad$ Flexibility Requirement}

To fulfill the requirement of flexibility, several global features must be present in the system:

- There should be a priority system for input streams, runs, or even files.

- Most, if not all, stages in the pipeline must be configurable before running and at run time.

- There should be a centralized sub-system to accept requests like change priority, add/remove stream, reprocess a run, ... etc. This sub-system should be smart enough to know how to re-configure the pipeline in response of the request. 


\section{Prototype and Mock Data Challenge}

CDF has been using a prototype Run 2 farm for over one year with great success. The prototype consists of 4 "I/O" nodes (dual PII/400's) with Gbit Ethernet interfaces, 512 MByte memory and large disk storage, 14 "worker" nodes (dual PII/400's) with 100 Mbit Ethernet interfaces, 512 MByte memory and 18 GByte local disk storage. This system has been used by the two Fermilab collider experiments CDF and D0, the Computing Division, and by lattice QCD collaborators. The prototype farm has been used to study the CDF executable behavior, the batch scheduling system, the CDF farms control software system, I/O rates and was used for the first Mock Data Challenge. Experience from this system was used in the specification of the first large purchase of worker and I/O nodes for Run 2.

Tests on the prototype showed that the system could easily scale from one to as many of 14 (the maximum available) nodes, consisting of 28 processors. The network and disk subsystems were tested and no serious limitations were found to the transfer of data. Given the disks available and the network connections, a measured rate of $20 \mathrm{MByte} / \mathrm{sec}$ from the 14 worker nodes to and from the I/O node was as expected.

The first Mock Data Challenge was performed to study the entire CDF offline system. The study was termed a "connectivity test" in that the main goal was to connect the various CDF offline systems for the first time and to run a modest number of simulated events through the system. The Challenge consisted of a simulated dataset of about 500,000 events, the level 3 trigger system, the data logging system, the data file catalog, the data handling software(2), a tape robotic system with tapedrives, and the prototype farm. The goal was to run all the systems simultaneously and to have the data move seamlessly from the level 3 systems through the farms and eventually onto separate physics streams, which would then be ready for analysis by physicists on the experiment. The first Mock Data Challenge was conducted between December 1999 and February 2000.

The second Mock Data Challenge (MDC2), to be run in late spring 2000, is meant to stress the systems at rates comparable to rates expected during data taking for a long enough period of time that bottlenecks, both serious and subtle, are identified and possibly fixed. Doing so will go a long way to preparing the offline systems, including the farms, for full data taking in spring of 2001 and extending for about 5-6 years after. 


\section{Scalability beyond Run 2}

The initial goals for Run 2 are accomplished with the L3 output rates (and consequent farm rates) of a maximum of $75 \mathrm{~Hz}$ (approximately $20 \mathrm{MByte} / \mathrm{s}$ ) and a DC rate of $28 \mathrm{~Hz}$. Though the exact parameters are not yet known it is clear that the luminosity will increase substantially after the first two years. It is assumed that the data-taking rate will also increase. The farms and production systems design should scale to substantially higher rates without a major redesign of the system. The following components should scale easily, mainly by improvements in computing technology.

- $\mathrm{CPU}$

The Pentium and follow-on commodity computing chips will continue to improve. This will automatically lead to increased CPU power as older PCs are replaced. In addition, the farm should scale with the addition of PCs. The system is modular enough that the increase in PCs can be quite large without reaching any serious bottlenecks or those bottlenecks can be eliminated by a simple change in topology somewhere else in the system.

- $\mathrm{I} / \mathrm{O}$

The increased I/O required through the system can be addressed in many ways. First, the tape I/O can be improved by using newer, faster tapedrives or by adding tapedrives. Disk I/O rates and disk storage requirements will increase and can be accommodated with expected improvements in disk and disk interface technology. The CPU necessary to split the data into final physics datasets can be increased by the upgrade and/or addition of processors to an I/O node or by adding a second (or third or fourth) I/O node to the system. The network can be made more capable by upgrading links from 100 Mbit to 1 Gbit or 1 Gbit to 10 Gbit or by using multiple interfaces. The farm switch can be replaced with a more capable switch or if that is not possible by adding a second switch.

In all of these cases the improvements required can be achieved because of the modular design of the system.

- Software

A more serious concern is the capability of the software system to manage the systems and to function properly with a large scale-up of the systems. There are a few places (e.g. database access) where the software is not modular and will require capabilities substantially greater than those achieved up to now or during the first two years of Run 2. This will have to be studied and understood before such an upgrade is attempted. The rest of the software is modular already and should have little or no trouble scaling substantially beyond that which will be achieved in Run 2 . 


\section{Conclusion}

Run 2 will produce large amounts of data which will have to be processed quickly. The ability to handle the data quickly and easily will have a direct impact on the ability for CDF to analyze the data and to produce physics results in a timely fashion. The system described in this paper does have the necessary software design and hardware and software implementation to keep up with Run 2 data rates. Further improvements to the system to scale up to expected increases in luminosity and data-taking rate are possible and will allow the production systems to function for many years.

\section{Acknowledgements}

This work is supported by the U.S. Department of Energy under contract No. DE-AC02-76CH03000, the National Science Council of the Republic of China, and the U.S.-Slovak Science and Technology Joint Fund under Project Number 00198,

\section{References}

[1] I. Mandrichenko, et al., Farm Batch System and Fermi Inter-Process Communications and Synchronization Toolkit, FERMILAB-Conf-00/083, April, 2000, published in the proceedings of the International Conference on Computing in High Energy and Nuclear Physics, Padova, Italy, Feb 7-11, 2000..

[2] T. Watts, et al., Overview of CDF Run II Data Handling System, published in the proceeding of the International Conference of Computing in High Energy and Nuclear Physics, Padova, Italy, Feb 7-11, 2000. 